The Astrophysical Journal, 463:382-390, 1996 May 20

(C) 1996. The American Astronomical Society. All rights reserved. Printed in U.S.A.

\title{
SEISMOLOGY OF THE STANDARD SOLAR MODEL: TESTS OF DIFFUSION AND THE OPAL AND THE MHD EQUATIONS OF STATE
}

\author{
D. B. GUENTHER \\ Department of Astronomy and Physics, Saint Mary's University, Halifax, NS, Canada B3H 3C3 \\ AND \\ Y.-C. Kim AND P. DemarQue \\ Center for Solar and Space Research, Department of Astronomy, Yale University, New Haven, CT 06520 \\ Received 1995 July 27; accepted 1995 December 4
}

\begin{abstract}
The OPAL equation-of-state tables and the MHD equation-of-state tables are utilized in the calculation of new standard solar models that also include helium diffusion. The structure of the models and their $l=0-100 p$-mode oscillation spectra are compared. The $p$-mode frequencies of the standard solar model are dramatically and nearly identically affected by the inclusion of these detailed equation-of-state formulations. The sensitivities of the $p$-mode frequencies to other physical uncertainties, in particular the surface conditions, are also discussed.
\end{abstract}

Subject headings: atomic data - diffusion - equation of state - Sun: interior - Sun: oscillations

\section{INTRODUCTION}

It is believed that the known uncertainties in the structure of the outer layers of the Sun (mixing-length approximation, atmospheric structure), the opacities, and the equation of state are responsible for most of the discrepancy between the frequencies of the observed $p$-modes and the frequencies of the $p$-modes calculated from the standard solar model (Bahcall \& Ulrich 1988; Christensen-Dalsgaard 1988; Cox, Guzik, \& Kidman 1989; Cox, Guzik, \& Raby 1990; Guzik \& Cox 1991; Guenther et al. 1992). Progress, though, is being made to improve the physics of the solar model in all three areas.

When high quality high-l $p$-mode observations become available it will be possible to probe the superadiabatic layer of the Sun in sufficient detail to seriously test various models of convection. Helioseismic tests at low and intermediate $l$-values by Paterno et al. (1993) of Canuto \& Mazzitelli's (1992) new formulation of mixing-length theory are encouraging, although the theoretical basis of the theory is still being debated. Solar models using Balmforth's (1992a, $\mathrm{b}, \mathrm{c}$ ) models of convection also appear to improve the low and intermediate $l$-valued $p$-mode frequencies. Currently, a number of solar modeling groups are attempting to include a more realistic description of convection in the solar model based on detailed 3-D numerical simulations of turbulent convection in the outer layers of the Sun.

With regard to the opacities, a great deal of progress has already been made. Several studies have revealed that solar models that employ the OPAL opacity library tables (Iglesias \& Rogers 1991; Rogers \& Iglesias 1994) have a significantly improved $p$-mode spectrum, when compared to solar models based on the LAOL (Los Alamos Opacity Library) (Heubner et al. 1977), the previous standard for astrophysical opacities. OPAL opacities differ from LAOL opacities by as much as a factor of three near $3 \times 10^{5} \mathrm{~K}$.

Not often mentioned but equally important with regard to improving the agreement between the observed and predicted $p$-mode frequencies has been the adoption of a much more accurate tabulation of the abundances of the elements in the Sun (Demarque \& Guenther 1991). The mixture of
Anders \& Grevesse (1989) (with meteoritic iron abundance) is significantly different from the Ross \& Aller mixture (1976) and the Cox \& Stewart mixture (1969) that were used in nearly all previous stellar model and opacity table constructions. The iron abundance in the Cox \& Stewart mixture, for example is an order of magnitude lower than in the Anders \& Grevesse mixture. The neon abundance in the Ross \& Aller mixture is a factor of 2 lower than in the Anders \& Grevesse mixture. Neon is important (Guenther \& Sarajedini 1988) because uncertainties in its abundance affect the opacity and equation of state in the temperature range near the base of the Sun's convection zone (Rogers, Swenson, \& Iglesias 1995). Neon is also important because its abundance is relatively high in the Sun, comparable to the abundance of nitrogen. Unfortunately, the abundance of neon (and the other noble gases) cannot be directly measured in the Sun and must be inferred from other sources.

The frequencies of acoustic modes depend on the run of the sound speed in the Sun, which in turn is related to the equation of state. Accompanying the OPAL opacities are the OPAL equation-of-state tables. Although, the OPAL equation-of-state tables have been available for some time, the grid resolution of the tables has not been fine enough to permit accurate determinations of the first and second order derivatives of the basic thermodynamic variables. Finer meshed tables are now available (Rogers 1995; Rogers, Swenson, \& Iglesias 1995).

The MHD equation-of-state tables (Mihalas, Däppen, \& Hummer 1988; Hummer \& Mihalas 1988) have been available for some time and have been used by several groups to construct solar models. The frequencies of nearly all of the $p$-modes are improved primarily because the outer ionization zones, located in the convection zone of the Sun are more accurately modeled by the MHD equation of state (Christensen-Dalsgaard, Däppen, \& Lebreton 1988; Kim, Demarque, \& Guenther 1991; Guenther et al. 1992). The MHD equation of state, though, may have some problems in the convective layers of the Sun (Rogers, Swenson, \& Iglesias 1995).

The primary purpose of this paper is to describe the seismological effects of the OPAL equation of state. The 
models that we have calculated which are discussed in this paper are the following:

ssm - a reference standard model - no diffusion and standard Saha based equation of state;

opal - a model that uses the OPAL equation-of-state tables;

mhd - a model that uses the MHD equation-of-state tables;

diff - a model that includes helium diffusion;

diff + opal - a model that includes helium diffusion and uses the OPAL equation-of-state tables;

diff + opal + kappa - a model that includes helium diffusion, uses the OPAL equation-of-state tables, and a uses a tweaked molecular opacity.

We show that the OPAL equation of state and the MHD equation of state increase the frequencies of all of the $p$-modes by $\sim 8 \mu \mathrm{Hz}$. We show that diffusion is also required to reduce the discrepancy between the models and the observations. In addition, we show that an opacity tweak to the surface layers can be used to eliminate the discrepancy in frequency spacing between the models and the observations. The frequencies of the $l=0-100 p$-modes of the model that includes diffusion, uses the new OPAL equation-of-state tables, and uses the tweaked opacities are within $\pm 3 \mu \mathrm{Hz}$ of the observed frequencies (corresponding to a discrepancy of $\sim 0.2 \%$ ).

In addition to monitoring the seismological effects of improvements to existing input physics, one must also consider the possibility that the existing physics are incomplete. To date, solar modelers have considered the effects of diffusion and rotation. The $p$-mode spectrum of solar models that include helium diffusion are distinct from those that do not. Most stellar diffusion calculations model the gravitational settling of helium in a hydrogen environment (Cox, Guzik, \& Kidman 1989; Proffitt \& Michaud 1991; Christensen-Dalsgaard, Proffitt, \& Thompson 1993; Guenther, Pinsonneault, \& Bahcall 1993; Guenther 1994). Some heavy element diffusion calculations have been attempted (Guzik \& Cox 1993; Proffitt 1994), but the effect on the structure does not appear to be as large. In addition, because the calculation of the diffusion coefficients is difficult for ions heavier than helium, the coefficients are far more uncertain. In previous studies we have found that diffusion reduces the $p$-mode discrepancy for some modes and increases it for others (Guenther 1994). We have also studied the effects of rotation, and the combined effects of diffusion and rotation on the structure and $p$-mode spectrum of the solar model (Chaboyer et al. 1995). Rotation does not affect the structure enough to perturb the $p$-mode frequencies. It does, though, inhibit the action of diffusion, equivalent to reducing the diffusion coefficients by a factor of 2 .

The next section summarizes the main discrepancies between the observed and calculated frequencies. Section 3 describes the model and pulsation calculations. The results are discussed in $\S 4$. Finally, $\S 5$ summarizes our findings.

\section{SYSTEMATICS OF THE DISCREPANCIES BETWEEN} OBSERVED AND CALCULATED FREQUENCIES

We, as others, have found it useful to compare the $p$-mode spectra of different solar models using a frequency difference plot, in which the frequencies of the $p$-modes of the model minus the frequencies of the Sun are plotted as a function of the observed frequencies. If the model and the Sun were a perfect match, all of the data.would lie along a horizontal line intercepting the frequency difference axis at $0 \mu \mathrm{Hz}$. For typical standard solar models the data are scattered up to $\pm 15 \mu \mathrm{Hz}$ about the $0 \mu \mathrm{Hz}$ line (corresponding to a discrepancy of $\sim 1 \%$ ).

The magnitude of the discrepancy (i.e., model frequency minus Sun frequency for each $p$-mode) depends on the frequency (or equivalently the order $n$ ) and the degree $(l)$ of the mode. To help distinguish different sources of error we define three distinct characteristics of the discrepancies as revealed in the frequency difference plots:

1. Slope error: the discrepancy increases with increasing frequency because the spacings between adjacent order $p$-mode frequencies of the model are greater than the observed spacings.

2. Offset error: the frequencies of the p-modes of the model are systematically lower than the observed frequencies (when the slope discrepancy is removed).

3. Thickness error: the spread or size of the discrepancies depends on the degree $l$ and the frequency of the $p$-modes (when the slope and offset discrepancy are removed), and is different for different modes.

An error in the slope corresponds to an error in the frequency spacing between $p$-modes adjacent in $n$, which is known from asymptotic theory (Tassoul 1980) to depend predominantly on the outermost layers of the Sun. The slope error is, therefore, probably a consequence of the uncertainties in the molecular opacities and the modeling of the transport of energy by convection and radiation in the outer layers. Because the $p$-modes are driven by turbulent convection in the superadiabatic layer, nonadiabatic corrections to the $p$-mode frequency calculation strongly affect the slope error. Guenther (1994), for example, has shown that the slope error is reduced by a factor of 2 when nonadiabatic effects due to radiation are taken into account in the $p$-mode frequency calculation. Balmforth $(1992 \mathrm{a}, \mathrm{b}, \mathrm{c})$ has also shown that the nonadiabatic effects due to turbulence have a similar effect.

The offset error and the thickness error are associated with internal structural differences between the model and the Sun. It is possible to reduce some of the discrepancies by tweaking the opacities (by less than $\pm 5 \%$ ) (Guzik \& Cox 1991 ) in specific regions of the Sun. In fact, opacity tweaks alone can produce models that reduce the $p$-mode frequency discrepancy to $\pm 5 \mu \mathrm{Hz}$ for $l=0-100$ modes. Note that because the OPAL opacities are estimated to be accurate to $\pm 25 \%$ (Rogers private communication) it is not unreasonable to expect that some of the discrepancies are a consequence of errors in the opacities. Because the opacities have no direct affect on the temperature gradients in the convective envelope, other errant physics, such as the equation of state, must also be responsible for the discrepancies. For example, solar models that utilize the MHD equationof-state tables, as noted above, almost completely remove the offset error.

\section{MODEL AND PULSATION CALCULATIONS}

The Yale Evolution Code (YREC; Guenther 1989, Pinsonneault et al. 1989), updated to include the OPAL (Rogers 1995; Rogers, Swenson, \& Iglesias 1995) and the MHD (Mihalas, Däppen, \& Hummer 1988; Däppen 1995) 
equation-of-state routines, was used to produce the solar models. We used the interpolation routines supplied with each equation-of-state, slightly modified so that total pressure is used as the independent variable.

The MHD equation-of-state tables are based on a single composition with the mixture ratio for $\mathrm{C}, \mathrm{N}, \mathrm{O}$ set equal to the C, N, O mixture ratio in the Ross-Aller mixture (Ross \& Aller 1976). Iron is padded to make $Z=0.02$. Full internal partition functions are used for $\mathrm{C}, \mathrm{N}, \mathrm{O}$ and all their ionic subspecies. In the case of $\mathrm{Fe}$, full partition functions are used for $\mathrm{Fe}$ and $\mathrm{Fe}^{+}$, while for the more highly ionized subspecies only ground states are included. The tables contain all the necessary thermodynamic variables and their derivatives.

The OPAL equation-of-state distribution includes several tables covering a range of $Z(0.00,0.02,0.04)$ and an interpolation program that is used to generate the final table at a specified $Z$. The mixture of heavy elements is scaled to the Anders \& Grevesse mixture (Anders \& Grevesse 1989) and includes all elements up to and including neon, with heavier elements being added to the abundance of neon. Note that unlike the opacity calculation, iron does not dominate the results of the elements heavier than neon (Rogers, Swenson, \& Iglesias 1995). All stages of ionization and excitation are calculated. As of this writing, some of the derivatives of the thermodynamic variables, required for pulsation studies, are not present in the OPAL tables. To obtain the missing data, we calculated the derivatives using finite difference formulae. We also tried substituting the derivatives calculated by our standard analytical equationof-state routines. The source of the derivatives should and does not have any effect on the structure of the models; in fact, the models were identical to seven significant digits in all physical variables.

YREC's original equation-of-state routines solve the Saha equation for hydrogen and helium below $T=2.0 \times 10^{6} \mathrm{~K}$, and assume full ionization above this temperature. The Debye-Hückel correction see Guenther et al. 1992) is applied above $T=1.0 \times 10^{6} \mathrm{~K}$. YREC uses the nuclear energy generation routines of Bahcall (Bahcall \& Pinsonneault 1992, 1993) and the cross sections listed in Bahcall's Neutrino Astrophysics (Bahcall 1989). The diffusion models were calculated using the Bahcall \& Loeb formulation and diffusion coefficients (Bahcall \& Loeb 1990).

All models were evolved from a zero-age main-sequence (ZAMS) model to the age of the Sun, 4.5 Gyr (Guenther $1989)$, in 50 equally spaced time steps. By adjusting the helium abundance and mixing length parameter of each model, the models were tuned to have identical radii to one part in $10^{7}\left(R_{\odot}=6.9598 \times 10^{10} \mathrm{~cm}\right)$ and identical luminosities to one part in $10^{6}\left(L_{\odot}=3.8515 \times 10^{33} \mathrm{ergs} \mathrm{s}^{-1}\right)$. To obtain numerically accurate $( \pm 0.2 \mu \mathrm{Hz}) p$-mode frequencies from the models, the models were forced to have approximately 1800 shells divided equally among the interior (inner $95 \%$ by radius of the model), the envelope (outer $5 \%$ by radius of the model), and the atmosphere (from the photosphere outward to the temperature minimum). OPAL opacities (Iglesias \& Rogers 1991) were used except at low temperatures where Kurucz opacities (Kurucz 1991) were substituted. We used the Anders \& Grevesse (1989) mixture of elements (with meteoritic iron abundance) and took the heavy element mass fraction for the standard solar model to be $Z=0.0188$ throughout. We also used this value of $Z$ for the MHD equation-of-state models, even though the MHD tables assume $Z=0.02$. This was done to maintain consistency with regard to the opacities which are more sensitive to the value of $Z$ than the equation-of-state. The atmosphere is modeled using the Krishna Swamy (1966) $T-\tau$ relation. This relation is derived from a fit to the Sun's observed $T-\tau$ dependence in the lower atmosphere.

YREC was modified to allow the opacity to be multiplied by a scaling factor, which itself is a function of temperature and density. For the tweaked opacity model we doubled the opacity at $\log T=3.8$, ramping the scaling factor up from 1 to 2 between $\log T=3.7$ and $\log T=3.8$, then ramping the scaling factor down from 2 to 1 between $\log T=3.8$ to $\log T=3.9$. Clearly, this is an ad hoc adjustment to the opacity.

The six models calculated are summarized in Table 1. The models were then input into Guenther's nonadiabatic nonradial stellar pulsation program (Guenther 1994). The pulsation program calculates the adiabatic and nonadiabatic frequencies of $p$-modes using the Henyey relaxation method. The nonadiabatic calculation includes the effects of radiation gains and losses but does not consider the effects of turbulence on the $p$-modes, which may be equally important (Balmforth 1992a, b, c). Nonadiabatic effects dominate in the superadiabatic layer, and hence, for a given $l$, the nonadiabatic adjustment to the frequency increases with increasing frequency of the mode.

\section{THE RESULTS}

\subsection{Structure Effects}

Some of the basic physical properties of the models are summarized in Table 1 . The models are identified in $\S 2$. The other columns list the mass fraction of hydrogen in the ZAMS model, the central mass fraction of hydrogen, the mass fraction of hydrogen at the surface, the metallicity, the base 10 logarithm of the central temperature and the central density $\left(\mathrm{g} \mathrm{cm}^{-3}\right)$, the fraction of mass contained in the convective envelope, the radius fraction depth of the base of the convective envelope, the neutrino capture rate for ${ }^{37} \mathrm{Cl}$ detectors and ${ }^{71} \mathrm{Ga}$ detectors, and the mixing length parameter.

All of the models are very similar in structure to the standard solar model. Diffusion has the most noticeable

TABLE 1

MODEL SUMmarY

\begin{tabular}{|c|c|c|c|c|c|c|c|c|c|c|c|}
\hline Model & $X_{\text {init }}$ & $X_{\text {core }}$ & $X_{\text {surf }}$ & $Z$ & $\log T_{\text {core }}$ & $\log \rho_{\text {core }}$ & $M_{\mathrm{cz}}$ & $x_{\mathrm{cz}}$ & ${ }^{37} \mathrm{Cl}$ & ${ }^{71} \mathrm{Ga}$ & $\alpha$ \\
\hline ssm. & 0.7107016 & 0.3602190 & 0.7107016 & 0.0188 & 7.19122056 & 2.176337 & 0.0208752 & 0.723 & 6.73 & 125 & 1.97614 \\
\hline diff . & 0.7118180 & 0.3552767 & 0.7286899 & 0.0188 & 7.19230297 & 2.181098 & 0.0231738 & 0.715 & 7.02 & 126 & 2.05048 \\
\hline mhd. & 0.7104347 & 0.3601651 & 0.7104347 & 0.0188 & 7.19124257 & 2.176447 & 0.0205116 & 0.725 & 6.74 & 125 & 1.94998 \\
\hline opal . & 0.7112328 & 0.3606535 & 0.7112328 & 0.0188 & 7.19111199 & 2.175911 & 0.0211992 & 0.722 & 6.70 & 126 & 1.93642 \\
\hline diff + opal & 0.7123486 & 0.3557050 & 0.7291485 & 0.0188 & 7.19219272 & 2.180672 & 0.0232644 & 0.714 & 6.99 & 126 & 2.00863 \\
\hline diff + opal + kappa.. & 0.7123435 & 0.3557421 & 0.7291702 & 0.0188 & 7.19218718 & 2.180639 & 0.0231898 & 0.715 & 6.98 & 126 & 2.41608 \\
\hline
\end{tabular}


affect on the values listed in the table. It reduces the surface abundance of helium, increases the central temperature and density, and, as a consequence, increases the total neutrino flux of the model. Our diffusion results are consistent with the results of other diffusion calculations (Cox, Guzik, \& Kidman 1989; Proffitt \& Michaud 1991). The MHD and OPAL equation-of-state have very little effect on the structural variables listed in Table 1.
To see the effects of diffusion, the OPAL equation of state and the MHD equation of state on the model itself we plot: the adiabatic exponent $\Gamma_{1}$ versus the radius fraction, $x$, in Figure $1 a$; the mean molecular weight, $\mu$, versus the radius fraction, in Figure 2; and the temperature gradient $\nabla$ versus the radius fraction, in Figure 3. In Figure $1 a$ we see that $\Gamma_{1}$ differs by less than $1 \%$ for the new equation-of-state calculations (OPAL and MHD) compared to the analytical

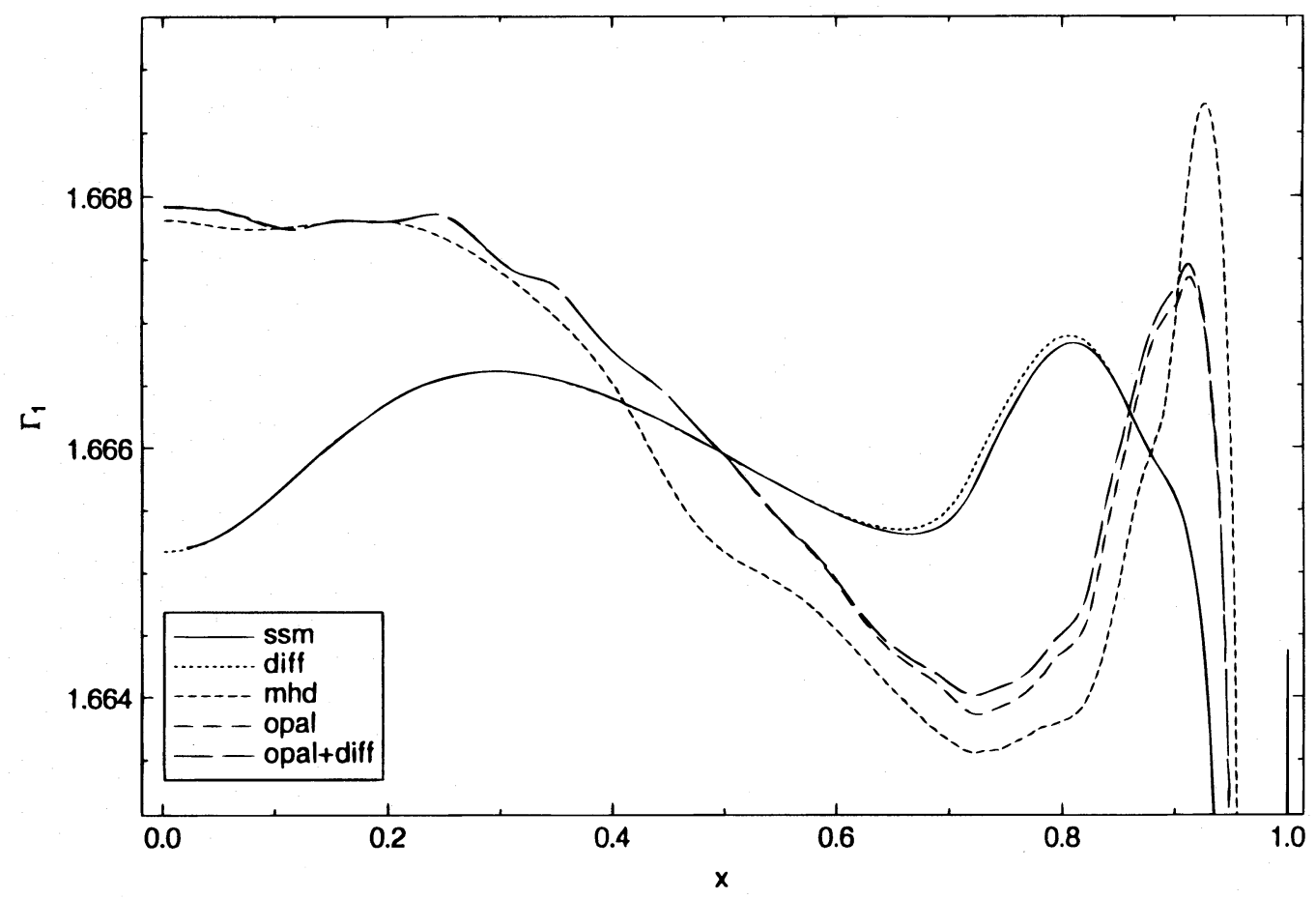

FIG. $1 a$

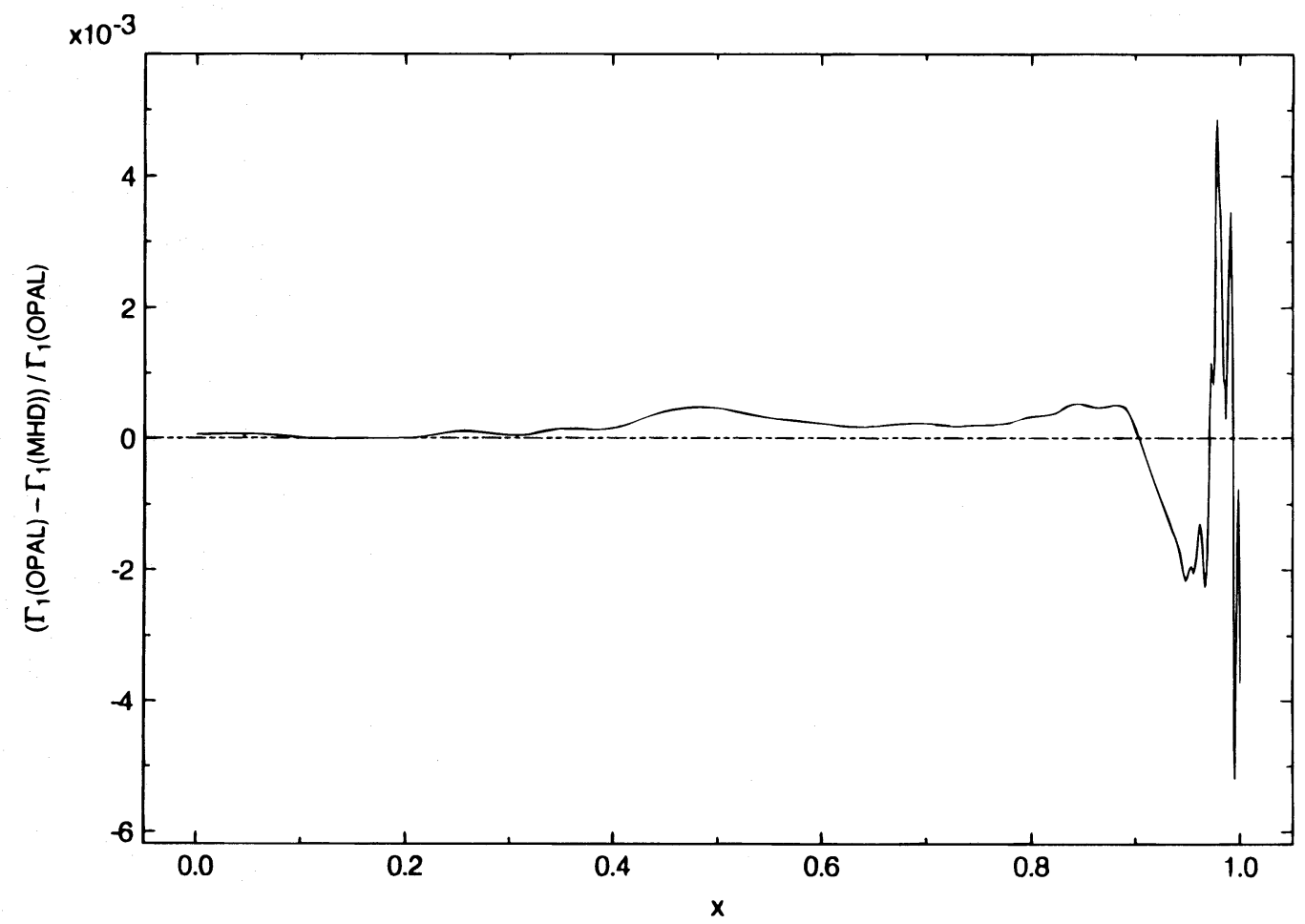

FIG. $1 b$

FIG. 1.-(a) Adiabatic exponent plotted opposite the radius fraction for the solar models indicated. The opal + diff + kappa model is not plotted because it overlaps the opal + diff model. (b) Fractional difference between the adiabatic exponents of the opal and the mhd model plotted opposite the radius fraction. 
equation-of-state used in YREC. In Figure $1 b$ we see that the MHD and OPAL equations of state are similar throughout the Sun except near the surface where the MHD equation of state exhibits what Rogers, Swenson, \& Iglesias (1995) call a "pressure spike," seen as dip near $x=0.94$. The differences just below the surface are associated with very slight differences in the location of the hydrogen and helium ionization regions in the two models. The pressure spike in the MHD equation of state is not believed to be physically real. The effect of diffusion on $\Gamma_{1}$ is not very large by comparison; the largest perturbation occurring in and just below the convection zone $\left(x_{\mathrm{cz}}=0.71\right)$.

In Figure 2, the effects of diffusion are more noticeable. The mean molecular weight is reduced in the convection zone of the diffusion models (diff and diff + opal) because helium has been drained out, settling toward the interior of the model. More curious is the sudden drop in the mean molecular weight at $x=0.73$ for the ssm and the diff models. This is an artifact of the cut-off temperature for the Saha calculation of the number density in YREC's equation-of-state routines. YREC uses the Saha equation to calculate the number density of hydrogen, helium, and the electrons below $T=3.0 \times 10^{6} \mathrm{~K}$. Above this temperature YREC assumes the plasma is completely ionized. The drop occurs at this temperature. Clearly, according to the Saha equation, not all of the plasma is completely ionized at this temperature. Note that the Saha equation cannot be used as one approaches the interior because pressure ionization becomes important. As the density increases, the ionization energy decreases, which limits the number of allowed bound states (see, e.g., Kippenhahn \& Weigert 1990). Again, as revealed by Figure 2, the OPAL and MHD equations of state appear to be similar throughout the Sun.

In Figure 3 we see the effect on the temperature gradient. Diffusion does not have any noticeable affect on the profile but does affect the location of the base of the convection zone, which is deepened by the effects of diffusion. From inversion of the $p$-mode frequencies Christensen-Dalsgaard, Gough, \& Thompson (1991) have determined the depth of the convection zone to be $x_{\mathrm{cz}}=0.713 \pm 0.003$. The three solar models that include diffusion have convection zone depths close to this value, the others have convection zones that are too shallow.

In Figure 4, a plot of the perturbations to the sound speed $\left(c_{\text {model }}-c_{\text {ssm }}\right) / c_{\text {ssm }}$ as a function of radius fraction, we see that diffusion does not affect the sound speed above the base of the convection zone. The OPAL and the MHD equation of state tables, on the other hand, do, and once again their effects are similar. This is not too surprising, since, as we have already noted, both the OPAL and MHD equation of state do improve the calculation of $\Gamma_{1}$ and $\mu$ (upon which the sound speed depends) in the convection zone. Notice, also, that below the base of the convection zone, diffusion increases the sound speed, whereas the OPAL and MHD equations of state decrease the sound speed.

\subsection{Seismology Effects}

In Figure 5 we show the frequency difference plots, model frequency minus observed frequency versus observed frequency, for $p$-modes with $l=1,2,3,4,10,20,30,40,50,60$, $70,80,90,100$. The lines connect common $l$-value $p$-modes. The thick lines correspond to $l=30,40$, and $50 p$-modes. These modes have inner turning points near the base of the convection zone, and are therefore sensitive to the structure near the base of the convection zone.

In Figure $5 a$ we see that the $p$-mode frequencies of the standard solar model are within $1 \%$ of the observed frequencies. The structure of the standard solar model is, therefore, already very close to the Sun's structure. However, one also notices some discrepancies in this kind of diagram. As pointed out in $\S 2$, the frequency differences do not lie on a horizontal line at $0 \mu \mathrm{Hz}$. The frequency differences depend on both $l$ and frequency. The frequency

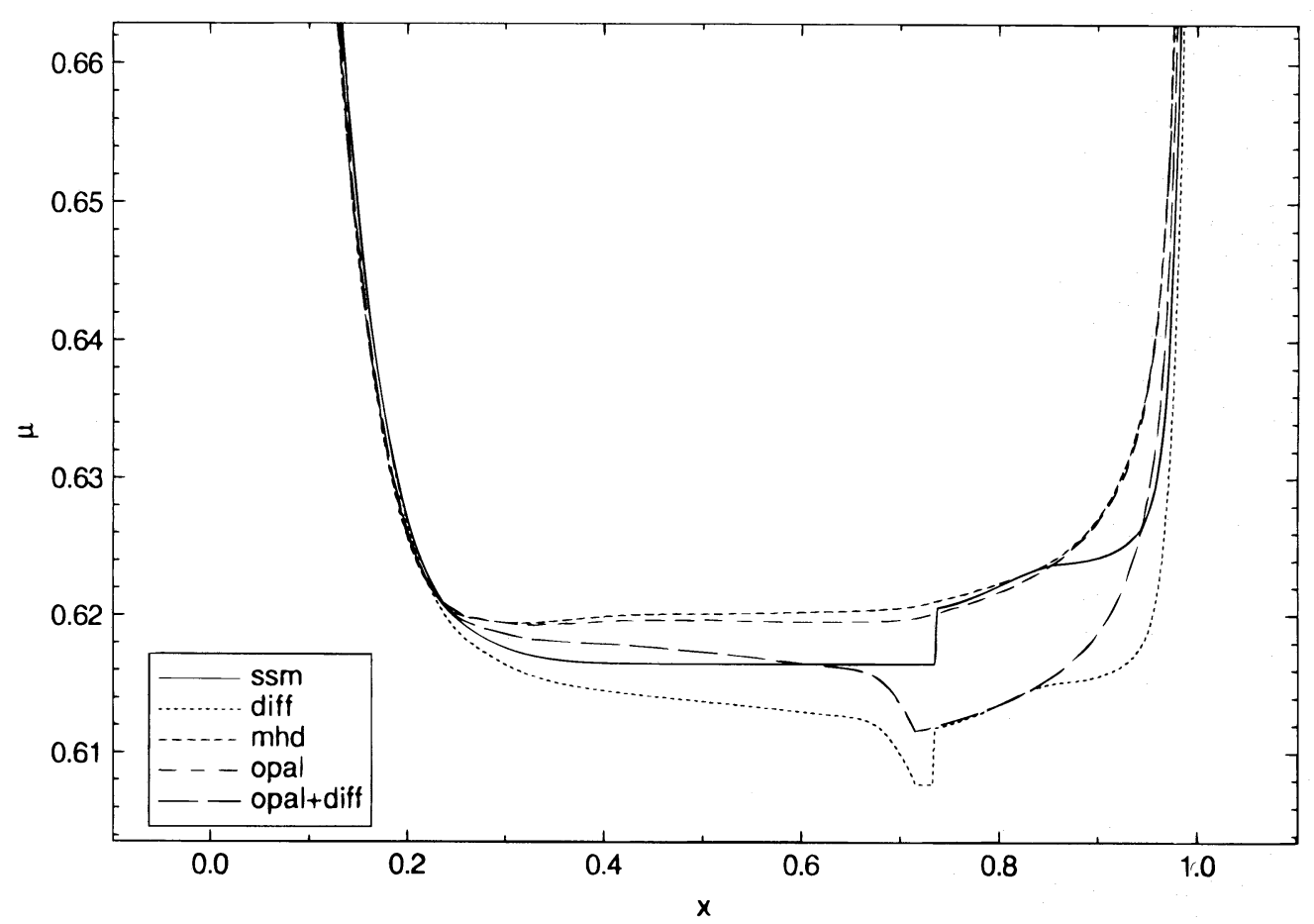

FIG. 2.-Mean molecular weight plotted opposite the radius fraction for the solar models indicated 


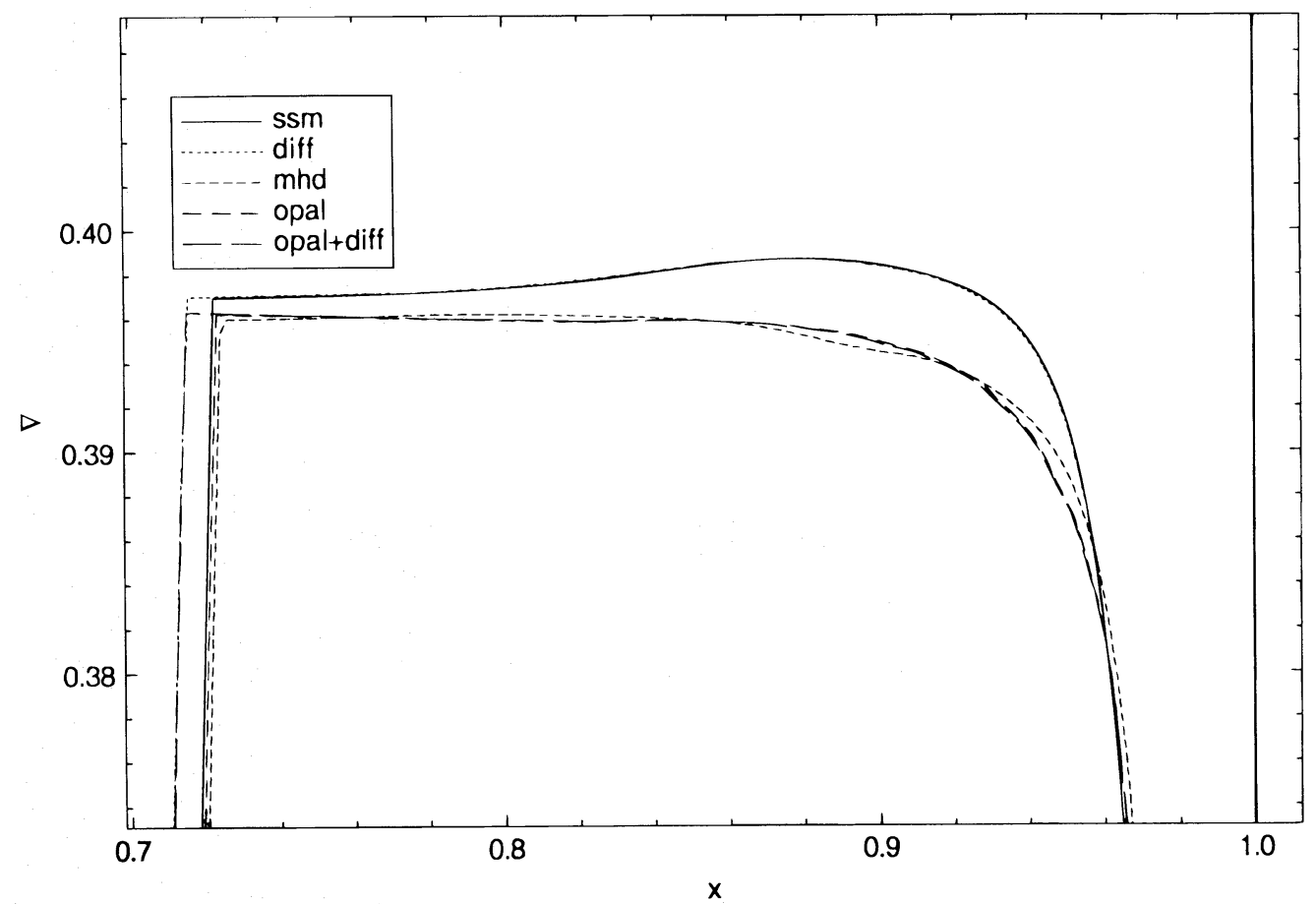

FIG. 3. - Temperature gradient plotted opposite the radius fraction, in the convection zone, for the solar models indicated

differences are too low, that is, the model frequencies averaged over the range plotted are $5-10 \mu \mathrm{Hz}$ too low (the offset error). The bundle of common $l$-value lines are spread apart by $\sim 5 \mu \mathrm{Hz}$, suggesting that the error in the structure of the model is not the same at all depths (the thickness error). Finally, the bundle of lines are not horizontal (the slope error). The slope of the lines directly depends on the separation in frequency between $p$-modes adjacent in $n$. The
" $n$-spacing" is sensitive to the very outermost layers of the Sun.

Figures $5 c$ and $5 d$ show the frequency differences for the solar models that include the MHD and OPAL equation of state, respectively. Their effects on the $p$-mode frequencies are similar, differing by only $\pm 0.5 \mu \mathrm{Hz}$. In addition, the MHD model results are consistent with the results of the earlier solar models in Kim et al. (1991) where it was noted

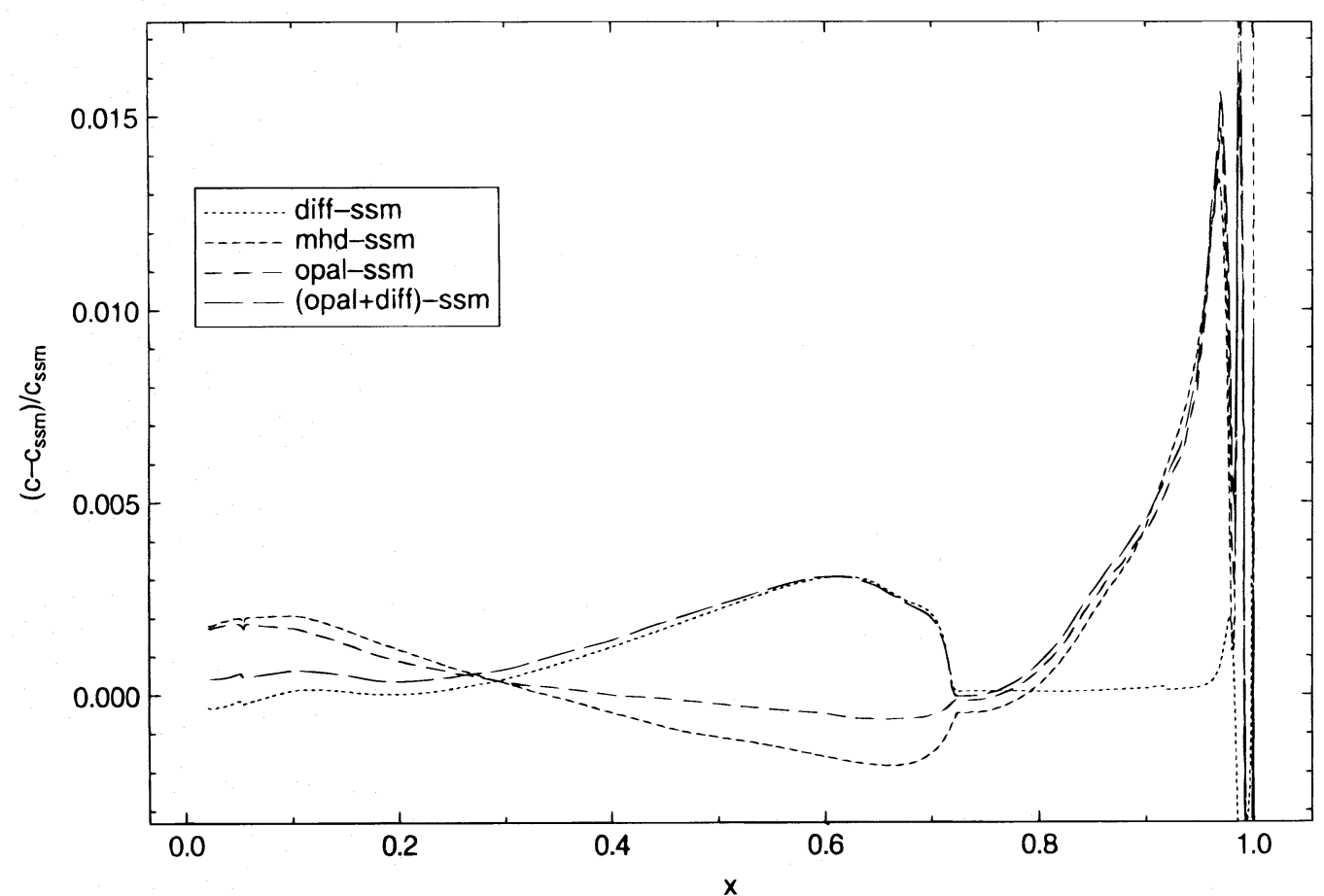

FIG. 4.-Relative sound-speed differences compared to the sound speed of the ssm model plotted opposite the radius fraction for the solar models indicated. 


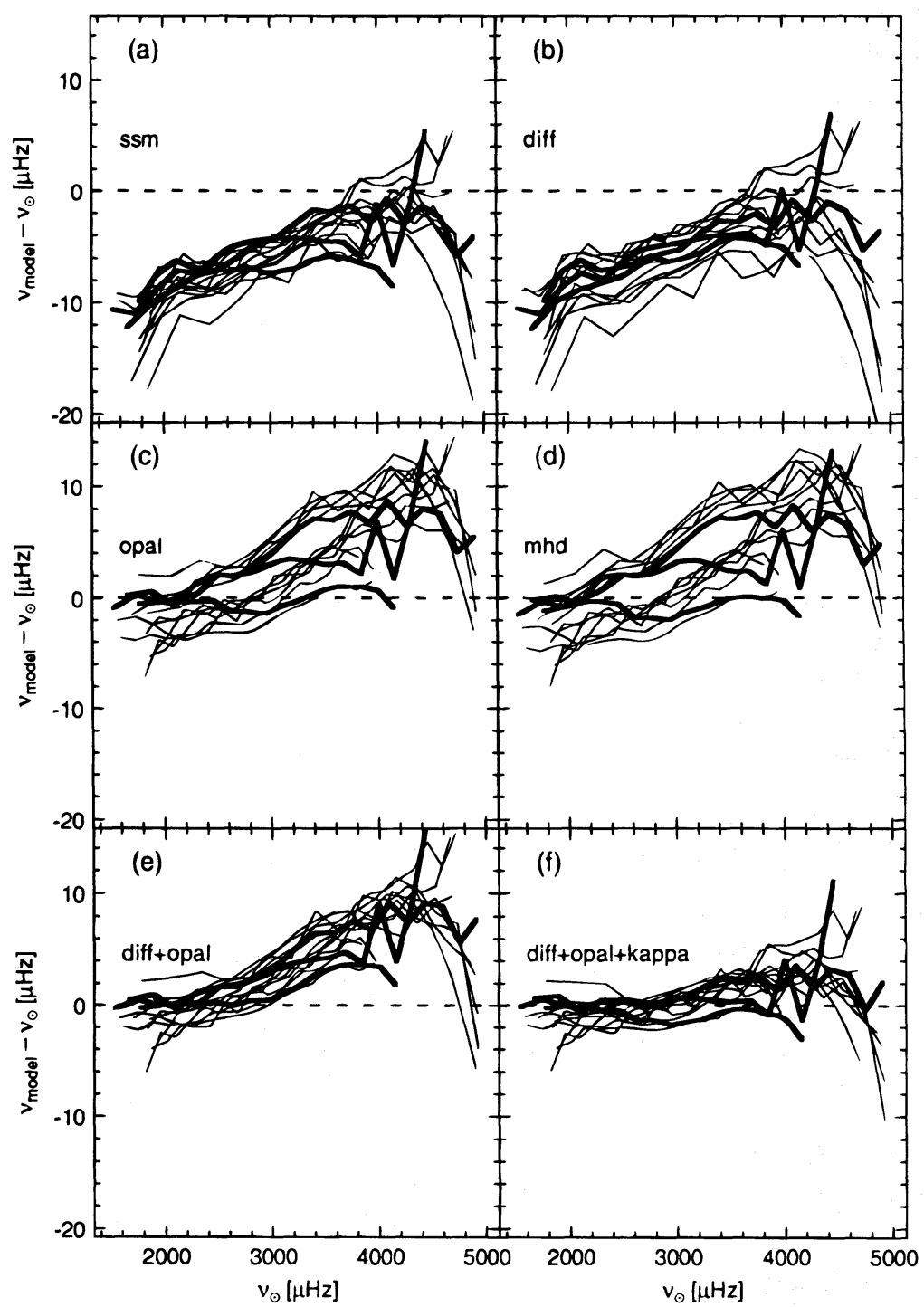

Fig. 5.-Frequency differences (model minus observed) plotted as a function of the observed frequencies for solar models indicated. Lines connect common $l$-valued $p$-modes for $l=0,1,2,3,10,20,30,40,50,60,70,80,90$, and 100 . Thisk lines identify the $l=30,40,50 p$-modes.

that the MHD equation of state does not improve the slope error (equivalent to $\Delta v$ in Fig. 7 of Kim et al.), but does increase the frequencies of the $p$-modes. This provides, to some extent, mutual verification of the OPAL and MHD equation-of-state tables. The modes of the opal and $m h d$ models are increased by $\sim 5-10 \mu \mathrm{Hz}$, which nicely removes the offset error. The slope error is unaffected. More problematic though, the thickness error is worsened. Although not identifiable in the plots, we note that the $l=0,1,2$, and $3 p$-modes lie along the bottom of the bundle of lines, and the $l=70,80,90$, and $100 p$-modes lie along the top of the bundle of lines, with the other $l$-values filling in the intermediate regions of the bundle of lines.

The diffusion model is shown in Figure $5 b$. The effect of diffusion on the $p$-mode frequencies is barely noticeable. Comparing the thick lines corresponding to $l=30,40,50$, one notices that the three lines are more tightly bundled together in the diffusion model than in the standard solar model. The other $l$-value lines appear to be relatively unaffected, although an examination of the data reveals that the high $l$-value lines are shifted downwards and the low $l$-value lines are shifted upwards. This is exactly the opposite of the MHD and OPAL equation-of-state models.
In Figure $5 e$ we show the frequency differences of the combined OPAL equation of state and diffusion model. Predictably, the thickness error is reduced, as the shifting of the high and low $l$-valued $p$-modes by the OPAL equationof-state and diffusion partially cancel each other out. Because diffusion combined with the OPAL equation-ofstate improves both the thickness error and the offset error, where separately they do not, we are led to believe that helium diffusion (or something that similarly affects the p-modes) must be included in the solar model calculation. Unfortunately, we still cannot conclude definitively that diffusion is occurring in the Sun because magnetic fields, seated near the base of the convection zone, may have an equally sizable and similar affect on the $p$-mode frequencies (Libbrecht \& Woodard 1990; Goldreich et al. 1991; Dziembowski \& Goode 1990, 1991; Lydon, Guenther, \& Sofia 1996).

It is possible that $p$-mode observations on other stars will enable us to settle this issue because the efficiency of helium diffusion is believed to depend sensitively on stellar mass. The $p$-mode observations of the two components of $\alpha$ Centauri, for example, would be very helpful: $\alpha$ Cen A $\left(1.1 M_{\odot}\right)$ is expected to undergo more helium diffusion than the Sun, 
while $\alpha$ Cen B $\left(0.9 M_{\odot}\right)$, which has a deeper convection zone, should be unaffected by diffusion (Edmonds et al. 1992).

To demonstrate how easy it is to correct the slope error by altering the structure of the outer most layers of the model we show, in Figure $5 f$, the frequency differences of a model that includes a minor perturbation to the low temperature opacities. The actual tweak shown here was derived by trial and error. Several models were calculated with the opacity increased over a small 0.2 dex range in temperature between $\log T=3.0$ and $\log T=4.0$. We immediately discovered that the slope error is very sensitive to the opacities in the temperature range $\log T=3.7$ to $\log T=3.9$, i.e., the temperature regime near the surface of the Sun. Further investigation revealed that perturbations to the opacities in this temperature range produce the biggest effect on the temperature gradients in the superadiabatic layer. This result to us suggests that either the structure of the superadiabatic layer is not being modeled correctly by the Böhm-Vitense mixing-length theory or our atmosphere model is not accurate enough to produce the correct superadiabatic structure. We are currently investigating both possibilities.

\subsection{Overview}

The frequency difference plots (Fig. 5) in conjunction with structure plots (Figs. 1-4) show clearly how diffusion and the OPAL (or MHD) equation of state combine to improve the solar model. We see that, when compared to the OPAL or MHD equations of state, YREC's equation-of-state routines do not do a very good job in determining either $\Gamma_{1}$ or the mean molecular weight $\mu$, especially in the ionization regions of the solar model. By using MHD or OPAL, the sound speed in the convection zone is increased (relative to the standard solar model), hence, the frequencies of all the modes that pass through this layer (true for all of the modes considered here) are also increased because, to first order:

$$
\frac{\Delta v}{v} \propto\left(\int_{r_{i}}^{R} \frac{d r}{c}\right)^{-1},
$$

where $R$ is the radius of the star, $r_{i}$ is the inner turning point, and $c$ is the sound speed. Neither the OPAL nor the MHD equation of state affects the depth of the convection zone. Diffusion, however, does affect the convection-zone depth. The $l=30,40,50$ modes, plotted with heavy lines in Figure 4 , have inner turning points near the base of the convection zone, and hence, are sensitive to the precise location of the base. The frequencies of these modes best match the observations for solar models that include diffusion.

More subtle are the combined affects of diffusion and the OPAL equation of state. When either diffusion of OPAL are used, the sound speed below the convection zone is perturbed (compared to the reference standard solar model). The effect on the $p$-modes is detrimental - the thickness error for most of the modes is worsened. From the sound-speed curves we see that below the base of the convection zone the OPAL equation of state has the opposite effect on the sound speed as diffusion. Combined, the two effects partially cancel each other out producing a soundspeed curve which is closer to the original standard solar model. Regardless, in the interior this appears to be a better match to the observations. Needless to say, we must not be driven exclusively by the desire to produce a solar model with the observed $p$-mode spectrum because such a model may have no basis in physical reality. The possible impact of other physical process not included in the models, e.g., a magnetic field, cannot be eliminated at this point.

\section{SUMMARY AND CONCLUSIONS}

We have examined the structure and seismology of solar models that include the new OPAL equation of state tables. We find that the effect of the OPAL equation of state on the structure of the solar model and the $p$-mode frequencies is similar to the effects of the MHD equation of state, except that the OPAL equation of state does not produce the pressure spike near the surface that the MHD equation of state does. The $p$-mode frequencies of solar models that include the OPAL equation of state are systematically shifted upwards by $\sim 5-10 \mu \mathrm{Hz}$. This effectively eliminates the offset error where the model frequencies modes are nearly all too low. By itself, the OPAL equation of state appears to worsen the thickness error. We find that by also including the effects of helium diffusion in the model, we can reduce the thickness error. Also related to this, only by including diffusion in the model can we increase the depth of the convection zone to that deduced by helioseismic inversion of the $p$-modes (Christensen-Dalsgaard et al. 1991). The frequencies of $p$-modes that are sensitive to the location of the base of the convection zone are improved.

Although, we, as others (e.g., Turck-Chièz \& Lopes 1993), have found it easy to remove the slope error by including a tweak to the molecular opacity near the temperature 6300 $\mathrm{K}$, we do not attach any physical significance to this tweak. However, because the opacity tweak does perturb the temperature gradients in the superadiabatic layer, we believe that this strongly suggests that the superadiabatic layer, itself, is not being modeled correctly, possibly because the Böhm-Vitense (1958) mixing length formulation, used by YREC to describe convective energy transport, is inadequate or possibly because our atmosphere, which provides the surface boundary condition, is not accurate enough.

With regard to matching the $p$-mode oscillation spectrum the best solar models include the latest nuclear cross sections of Bahcall, the OPAL equation of state, the OPAL opacities at high temperatures, the Anders \& Grevesse mixture of elements, a model atmosphere fit to the Sun, and the effects of helium diffusion. We are suspicious of our atmosphere model and our modeling of convection using the Böhm-Vitense mixing-length theory. Our own tests of models with heavy element diffusion (Pinsonneault \& Guenther 1995) corroborate earlier work by Guzik \& Cox (1993), and show that heavy element diffusion has so little affect on oscillation frequencies $(<\sim 1 \mu \mathrm{Hz})$ that it is not yet possible, considering uncertainties in $Z$ and the existence of an overshoot layer, to state that heavy element diffusion is occurring in the Sun or that we are modeling it correctly. We are currently investigating solar models that include alternate forms of mixing-length theory, some of which are based on our three-dimensional simulations of solar convection (Kim et al. 1995, 1996). As the improved agreement between the observed and calculated frequencies attest, the best solar models today continue to step closer to the actual Sun, and the $p$-mode frequencies continue to help us decide where to step next.

The authors gratefully acknowledge support for this research from NSERC (D. B. G.), and from NASA grants NAG5-1486 and NAG5-2795 (P. D). 
Anders, E., \& Grevesse, N. 1989, Geochim. Cosmochim. Acta, 53, 197

Bahcall, J. N. 1989, Neutrino Astrophysics (Cambridge: Cambridge Univ. Press)

Bahcall, J. N., \& Loeb, A. 1990, ApJ, 360, 267

Bahcall, J. N., \& Pinsonneault, M. H. 1992, Rev. Mod. Phys., 64, 885

1993, ApJ, 395, L119

Bahcall, J. N., \& Ulrich, R. K. 1988, Rev. Mod. Phys., 60, 297

Balmforth, N. J. 1992a, MNRAS, 255, 603 .1992b, MNRAS, 255, 632

1992c, MNRAS, 255, 639

Böhm-Vitense, E. 1958, Z. Astrophys., 46, 108

Canuto, V. M., \& Mazzitelli, I. 1992, ApJ, 389, 724

Chaboyer, B., Demarque, P., Guenther, D. B., \& Pinsonneault, M. H. 1995, ApJ, 446, 435

Christensen-Dalsgaard, J. 1988, ESA SP-286, Proc. Symp. Seismology of the Sun and Sun-like Stars (Noordwijk: ESA), 431

Christensen-Dalsgaard, J., Däppen, W., \& Lebreton, Y. 1988, Nature, 336, 634

Christensen-Dalsgaard, J., Gough, D. O., \& Thompson, M. J. 1991, ApJ, 378,413

Christensen-Dalsgaard, J., Proffitt, C. R., \& Thompson, M. J. 1993, ApJ, 403, L75

Cox, A. N., Guzik, J. A., \& Kidman, R. B. 1989, ApJ, 342, 1187

Cox, A. N., Guzik, J. A., \& Raby, S. 1990, ApJ, 353, 698

Cox, A. N., \& Stewart, J. N. 1969, Radiative and Conductive Opacities for Twenty-three Stellar Mixtures, Nauchny Inf., 15, 3

Däppen, W. 1975, private communication

Demarque, P., \& Guenther, D. B. 1991, in Solar Interior and Atmosphere, ed. A. Cox, W. Livingston, \& S. Mathews (Tucson: Univ. of Arizona Press), 1186

Dziembowski, W., \& Goode, P. 1990, ApJ, 347, 540 .1991, ApJ, 376, 782

Edmonds, P., Cram, L., Demarque, P., Guenther, D. B., \& Pinsonneault, M. H. 1992, ApJ, 394, 313

Goldreich, P., Murray, N., Willette, G., \& Kumar, P. 1991, ApJ, 370, 752

Guenther, D. B. 1989, ApJ, 339, 1156
Guenther, D. B. 1994, ApJ, 442, 40

Guenther, D. B., Demarque, P., Kim, Y.-C., \& Pinsonneault, M. H. 1992, ApJ, 387, 372

Guenther, D. B., Pinsonneault, M. H., \& Bahcall, J. N. 1993, ApJ, 418, 469

Guenther, D. B., \& Sarajedini, A. 1988, ApJ, 327, 993

Guzik, J. A., \& Cox, A. N. 1991, ApJ, 381, 333 .1993, ApJ, 411, 394

Huebner, W. F., Merts, A. L., Magee, N. H., \& Argo, M. F. 1977, Los Alamos Scientific Laboratory Report, No. LA-6760-M

Hummer, D. G., \& Mihalas, D. 1988, ApJ, 331, 794

Iglesias, C. A., \& Rogers, F. J. 1991, ApJ, 371, 408

Kim, Y.-C., Demarque, P., \& Guenther, D. B. 1991, ApJ, 378, 407

Kim, Y.-C., Fox, P. A., Demarque, P., \& Sofia, S. 1996, ApJ, in press

Kim, Y.-C., Fox, P. A., Sofia, S., \& Demarque, P. 1995, ApJ, 442, 421

Kippenhahn, R., \& Weigert, E. 1990, in Stellar Structure and Evolution, (Berlin: Springer), 11

Krishna Swamy, K. S. 1966, ApJ, 145, 174

Kurucz, R. L. 1991, in Stellar Atmospheres: Beyond Classical Models, ed. L. Crivellari, I. Hubeny, \& D. G. Hummer (Dordrecht: Kluwer), 440

Libbrecht, K., \& Woodard, M. 1990, Nature, 345, 779

Lydon, T. J., Guenther, D. B., \& Sofia, S. 1996, ApJ, in press

Mihalas, D., Däppen, W., \& Hummer, D. G. 1988, ApJ, 331, 815

Paterno, L., Ventura, R., Canuto, V. M., \& Mazzitelli, I. 1993, ApJ, 402, 733

Pinsonneault, M. H., \& Guenther, D. B. 1995, private communication

Pinsonneault, M. H., Kawaler, S. D., Sofia, S., \& Demarque, P. 1989, ApJ, 338,424

Proffitt, C. R. 1994, ApJ, 425, 849

Proffitt, C. R., \& Michaud, G. 1991, ApJ, 380, 238

Rogers, F. J. 1995, private communication

Rogers, F. J., \& Iglesias, C. A. 1994, Science, 263, 50

Rogers, F. J., Swenson, F. J., \& Iglesias, C. A. 1995, ApJ, submitted

Ross, J. E., \& Aller, L. H. 1976, Science, 191, 1223

Tassoul, M. 1980, ApJS, 43, 469

Turck-Chièz, S., \& Lopes, J. 1993, ApJ, 408, 347 